\title{
Reexamination of Hagen-Poiseuille flow: Shape dependence of the hydraulic resistance in microchannels
}

\author{
Mortensen, Asger; Okkels, Fridolin; Bruus, Henrik
}

Published in:

Physical Review E

Link to article, DOI:

10.1103/PhysRevE.71.057301

Publication date:

2005

Document Version

Publisher's PDF, also known as Version of record

Link back to DTU Orbit

Citation (APA):

Mortensen, A., Okkels, F., \& Bruus, H. (2005). Reexamination of Hagen-Poiseuille flow: Shape dependence of the hydraulic resistance in microchannels. Physical Review E, 71(5), 057301.

https://doi.org/10.1103/PhysRevE.71.057301

\section{General rights}

Copyright and moral rights for the publications made accessible in the public portal are retained by the authors and/or other copyright owners and it is a condition of accessing publications that users recognise and abide by the legal requirements associated with these rights.

- Users may download and print one copy of any publication from the public portal for the purpose of private study or research.

- You may not further distribute the material or use it for any profit-making activity or commercial gain

- You may freely distribute the URL identifying the publication in the public portal

If you believe that this document breaches copyright please contact us providing details, and we will remove access to the work immediately and investigate your claim. 


\title{
Reexamination of Hagen-Poiseuille flow: Shape dependence of the hydraulic resistance in microchannels
}

\author{
Niels Asger Mortensen, Fridolin Okkels, and Henrik Bruus \\ MIC, Department of Micro and Nanotechnology, Bldg. 345 east, Technical University of Denmark, DK-2800 Kgs. Lyngby, Denmark
}

(Received 2 December 2004; published 5 May 2005)

\begin{abstract}
We consider pressure-driven, steady-state Poiseuille flow in straight channels with various cross-sectional shapes: elliptic, rectangular, triangular, and harmonic-perturbed circles. A given shape is characterized by its perimeter $\mathcal{P}$ and area $\mathcal{A}$ which are combined into the dimensionless compactness number $\mathcal{C}=\mathcal{P}^{2} / \mathcal{A}$, while the hydraulic resistance is characterized by the well-known dimensionless geometrical correction factor $\alpha$. We find that $\alpha$ depends linearly on $\mathcal{C}$, which points out $\mathcal{C}$ as a single dimensionless measure characterizing flow properties as well as the strength and effectiveness of surface-related phenomena central to lab-on-a-chip applications. This measure also provides a simple way to evaluate the hydraulic resistance for the various shapes.
\end{abstract}

DOI: 10.1103/PhysRevE.71.057301

PACS number(s): 47.60. $+\mathrm{i}, 47.10 .+\mathrm{g}$

\section{INTRODUCTION}

The rapid development in the field of lab-on-a-chip systems during the past decade has put emphasis on studies of shape dependence in microfluidic channels. Traditionally, capillary tubes would have circular cross sections, but today microfabricated channels have a variety of shapes depending on the fabrication technique in use. Examples are rectangular channels obtained by hot embossing in polymer wafers, semi-circular channels in isotropically etched surfaces, triangular channels in $\mathrm{KOH}$-etched silicon crystals, Gaussianshaped channels in laser-ablated polymer films, and elliptic channels in stretched polydimethylsiloxane (PDMS) devices (see, e.g., Ref. [1]).

The pressure-driven, steady-state flow of a liquid through long, straight, and rigid channels of any constant crosssectional shape is referred to as Hagen-Poiseuille (or simply Poiseuille) flow, and it is often characterized by the hydraulic resistance, $R_{\text {hyd }}=\Delta p / Q$, where $\Delta p$ is the pressure drop along the channel and $Q$ is the flow rate through the channel. In Fig. 1 is shown an arbitrarily shaped cross-section $\Omega$ in the $x y$ plane for a straight channel placed along the $z$ axis. A natural unit for the hydraulic resistance is given by dimensional analysis as $R_{\text {hyd }}^{*} \equiv \eta L / \mathcal{A}^{2}$, where $L$ is the channel length, $\eta$ the dynamic viscosity of the liquid, and $\mathcal{A}$ $=\int_{\Omega} d x d y$ the cross-sectional area. Typically, the fluid flow is subject to a no-slip boundary condition at the walls $\partial \Omega$ and thus the actual hydraulic resistance will depend on the perimeter as well as the cross-section area. This dependence can therefore be characterized by the dimensionless geometrical correction factor $\alpha$ given by

$$
\alpha \equiv \frac{R_{\mathrm{hyd}}}{R_{\mathrm{hyd}}^{*}} .
$$

In lab-on-a-chip applications [1,2], where large surface-tovolume ratios are encountered, the problem of the bulk Poiseuille flow is typically accompanied by other surface-related physical or biochemical phenomena in the fluid. The list of examples includes surface chemistry, DNA hybridization on fixed targets, catalysis, interfacial electrokinetic phenomena such as electro-osmosis, electrophoresis, and electro-viscous effects, as well as continuous edge-source diffusion. Though the phenomena are of very different nature, they have at least one thing in common; they are all to some degree surface phenomena and their strength and effectiveness depends strongly on the surface-to-volume ratio. It is common to quantify this by the dimensionless compactness $\mathcal{C}$ given by

$$
\mathcal{C} \equiv \frac{\mathcal{P}^{2}}{\mathcal{A}},
$$

where $\mathcal{P} \equiv \int_{\partial \Omega} d \ell$ is the perimeter of the boundary $\partial \Omega$ confining the fluid (see Fig. 1). For other measures of $\mathcal{C}$ we refer to Ref. 3 and references therein. In this paper we demonstrate a simple dependence of the geometrical correction factor $\alpha$ on the compactness $\mathcal{C}$ and our results thus point out a unified dimensionless measure of flow properties as well as the strength and effectiveness of surface-related phenomena central to lab-on-a-chip applications. Furthermore, our results allow for an easy evaluation of the hydraulic resistance for elliptical, rectangular, and triangular cross-sections with the geometrical measure $\mathcal{C}$ being the only input parameter. Above we have emphasized microfluidic flows because here a variety of shapes are frequently encountered. However, our results are generally valid for all laminar flows.

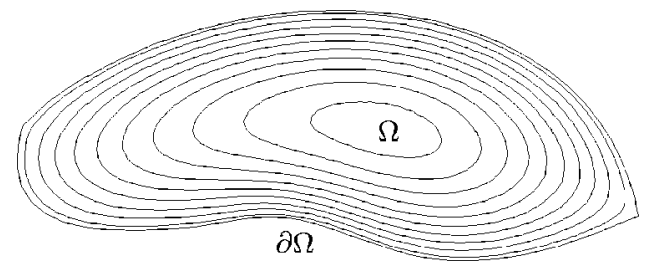

FIG. 1. An arbitrary cross-sectional shape $\Omega$ with boundary $\partial \Omega$ of a straight fluid channel with pressure-driven steady-state flow. The contours show the velocity $v(x, y)$ obtained numerically from Eq. (3) by a finite-element method. The velocity is zero at the boundary and maximal near the center-of-mass. 


\section{POISEUILLE FLOW}

Due to translation invariance along the $z$ axis the velocity field of a Newtonian fluid in a straight channel is parallel to the $z$ axis, and takes the form $\mathbf{v}=v(x, y) \mathbf{e}_{z}$. Consequently, the nonlinear term in the Navier-Stokes equation drops out [4], and, in steady state, given the pressure gradient $-(\Delta p / L) \mathbf{e}_{z}$, the velocity $v(x, y)$ is thus given by the Poisson equation,

$$
\left(\partial_{x}^{2}+\partial_{y}^{2}\right) v(x, y)=\frac{\Delta p}{\eta L},
$$

with the velocity being subject to a no-slip condition at the boundary $\partial \Omega$. The relation between the pressure drop $\Delta p$, the velocity $v(x, y)$, and the geometrical correction factor $\alpha$ becomes

$$
\Delta p=R_{\text {hyd }} Q=\alpha R_{\text {hyd }}^{*} Q=\alpha R_{\text {hyd }}^{*} \int_{\Omega} d x d y v(x, y),
$$

where $Q$ is the volume flow rate.

\section{THE GEOMETRICAL CORRECTION FACTOR VERSUS COMPACTNESS}

Our main objective is to find the relation between the geometrical correction factor $\alpha$ and the compactness $\mathcal{C}$ for various families of geometries.

\section{A. Elliptical cross section}

The elliptical family of cross sections is special in the sense that Eq. (3) can be solved analytically (see, e.g., Ref. [4]) and we can get an explicit expression for the geometrical correction factor introduced in Eq. (1). For an ellipse centered at the origin with semi-major and minor axes $a$ and $b$ it can be verified by direct insertion that

$$
v(x, y)=\frac{\Delta p}{\eta L} \frac{(a b)^{2}}{2\left(a^{2}+b^{2}\right)}\left(1-\frac{x^{2}}{a^{2}}-\frac{y^{2}}{b^{2}}\right)
$$

fulfills Eq. (3). From Eq. (4) it can now be shown that

$$
\alpha(\gamma)=4 \pi\left(\gamma+\gamma^{-1}\right)
$$

where $\gamma=a / b$. Furthermore, for an ellipse we have

$$
\mathcal{C}(\gamma)=\frac{16}{\pi} \gamma\left(\int_{0}^{\pi / 2} d \theta \sqrt{1-\left(1-\gamma^{-2}\right) \sin ^{2} \theta}\right)^{2} .
$$

The relation between $\alpha$ and $\mathcal{C}$ can now be investigated through a parametric plot. In order to get an approximate expression for $\alpha(\mathcal{C})$ we begin by inverting Eq. (6). By selecting the proper root we get $\gamma(\alpha)$ which we then substitute into Eq. (7) such that

$$
\mathcal{C}(\alpha)=\frac{1}{2 \pi^{2}}\left(\int_{0}^{\pi} d \theta \sqrt{\alpha+\sqrt{\alpha^{2}-(8 \pi)^{2}} \cos \theta}\right)^{2} .
$$

Expanding around $\alpha=8 \pi$ and inverting we get

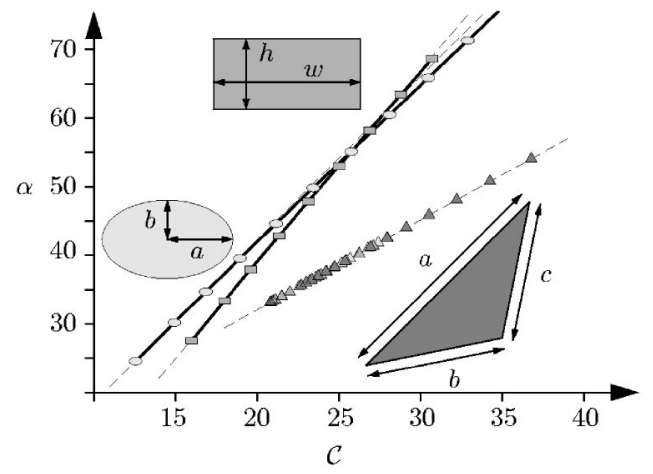

FIG. 2. The correction factor versus compactness for the elliptical, rectangular, and triangular classes. The solid lines are the exact results, and the dashed lines indicate Eqs. (9), (14), and (15). Numerical results from a finite-element simulation are also included $(\bigcirc, \square$, and $\triangle)$. Note that in the case of triangles all classes (right, isosceles, and acute/obtuse scalene triangles - marked by different grayscale triangles) fall on the same straight line.

$$
\alpha(\mathcal{C})=\frac{8}{3} \mathcal{C}-\frac{8 \pi}{3}+\mathcal{O}\left([\mathcal{C}-4 \pi]^{2}\right)
$$

and in Fig. 2 we compare the exact solution (solid line), from a parametric plot of Eqs. (6) and (7), to the approximate result (dashed line) in Eq. (9). Results of a numerical finiteelement solution of Eq. (3) are also included ( $\bigcirc$ points). As seen, there is a close-to-linear dependence of $\alpha$ on $\mathcal{C}$ as described by Eq. (9).

\section{B. Rectangular cross section}

For a rectangle with width-to-height ratio $\gamma=w / h$ we solve Eq. (3) using Fourier series [5]

$$
\begin{aligned}
v(x, y)= & \frac{\Delta p}{\eta L} \frac{4 h^{2}}{\pi^{3}} \\
& \times \sum_{n=1,3,5, \ldots}^{\infty} \frac{1}{n^{3}}\left(1-\frac{\cosh (n \pi x / h)}{\cosh (n \pi w / 2 h)}\right) \sin (n \pi y / h)
\end{aligned}
$$

is indeed a solution. Here, the coordinate system is chosen so that $-w / 2<x<w / 2$ and $0<y<h$. From Eq. (4) it follows that

$$
\alpha(\gamma)=\frac{\pi^{3} \gamma^{2}}{8}\left(\sum_{n=1,3,5, \ldots}^{\infty} \frac{n \gamma}{\pi n^{5}}-\frac{2}{\pi^{2} n^{5}} \tanh (n \pi \gamma / 2)\right)^{-1}
$$

and for the compactness we have

$$
\mathcal{C}(\gamma)=8+4 \gamma+4 / \gamma
$$

Using that $\tanh (x) \simeq 1$ for $x \gg 1$ we get

$$
\alpha(\gamma) \simeq \frac{12 \pi^{5} \gamma^{2}}{\pi^{5} \gamma-186 \zeta(5)}, \quad \gamma \gg 1
$$

and substituting $\gamma(\mathcal{C})$ into this expression and expanding around $\mathcal{C}(\gamma=2)=18$ we get 


$$
\alpha(\mathcal{C}) \approx \frac{22}{7} \mathcal{C}-\frac{65}{3}+\mathcal{O}\left([\mathcal{C}-18]^{2}\right)
$$

For the two Taylor coefficients we have used the first three terms in the continued fraction. In Fig. 2 we compare the exact solution, obtained by a parametric plot of Eqs. (11) and (12), to the approximate result, Eq. (14). Results of a numerical finite-element solution of Eq. (3) are also included ( $\square$ points). As in the elliptical case, there is a close-to-linear dependence of $\alpha$ on $\mathcal{C}$ as described by Eq. (14).

\section{Triangular shape}

For the equilateral triangle it can be shown analytically that $\alpha=20 \sqrt{3}$ and $\mathcal{C}=12 \sqrt{3}$ (see, e.g., Ref. [4]). However, in the general case of a triangle with side lengths $a, b$, and $c$ we are referred to numerical solutions of Eq. (3). In Fig. 2 we show numerical results ( $\triangle$ points), from finite-element simulations, for scaling of right triangles, isosceles triangles, and acute/obtuse scalene triangles (for the definitions we refer to Ref. [6]). The dashed line shows

$$
\alpha(\mathcal{C})=\frac{25}{17} \mathcal{C}+\frac{40 \sqrt{3}}{17},
$$

where the slope is obtained from a numerical fit and subsequent use of the first three terms in the continued fraction of this value. As seen, the results for different classes of triangles fall onto the same straight line. Since we have

$$
\mathcal{C}(a, b, c)=\frac{8(a+b+c)^{2}}{\sqrt{\frac{1}{2}\left(a^{2}+b^{2}+c^{2}\right)^{2}-\left(a^{4}+b^{4}+c^{4}\right)}}
$$

the result in Eq. (15) allows for an easy evaluation of $R_{\text {hyd }}$ for triangular channels.

\section{Harmonically perturbed circle}

By use of shape perturbation theory it is possible to extend the analytical results for Poiseuille flow beyond the few cases of regular geometries that we have treated above. In shape perturbation theory the starting point is an analytically solvable case, which then is deformed slightly characterized by some small perturbation parameter $\epsilon$. As illustrated in Fig. 3 the unperturbed shape is described by parametric coordinates $(\tilde{x}, \tilde{y})$ in Cartesian form or $(\rho, \theta)$ in polar form. The coordinates of the physical problem we would like to solve are $(x, y)$ in Cartesian form and $(r, \phi)$ in polar form.

As a concrete example we take the harmonic perturbation of the circle defined by the transformation

$$
\begin{gathered}
\phi=\theta, \\
r=a \rho[1+\epsilon \sin (k \theta)], \\
x(\rho, \theta)=a \rho[1+\epsilon \sin (k \theta)] \cos \theta, \\
y(\rho, \theta)=a \rho[1+\epsilon \sin (k \theta)] \sin \theta,
\end{gathered}
$$

where $a$ is length scale, $k$ is an integer $(>2)$ defining the order of the harmonic perturbation, $0 \leqslant \theta \leqslant 2 \pi$, and $0 \leqslant \rho$
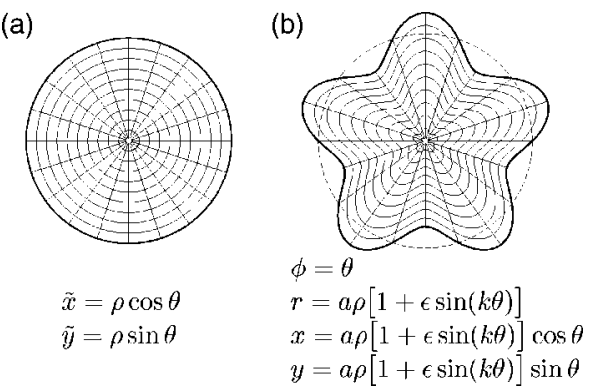

FIG. 3. (a) The geometry of the unperturbed and analytically solvable cross section, the unit circle, described by coordinates $(\tilde{x}, \tilde{y})$ or $(\rho, \theta)$. (b) The geometry of the perturbed cross section described by coordinates $(x, y)$ or $(r, \phi)$ and the perturbation parameter $\epsilon$. Here $a=1, k=5$, and $\epsilon=0.2$.

$\leqslant 1$. For $\epsilon=0$ the shape is unperturbed. The boundary of the perturbed shape is described by fixing the unperturbed coordinate $\rho=1$ and sweeping in $\theta$,

$$
\partial \Omega:(x, y)=(x[1, \theta], y[1, \theta]) .
$$

It is desirable to formulate the perturbed Poiseuille problem using the unperturbed coordinates. To obtain analytical results it is important to make the appearance of the perturbation parameter explicit. When performing a perturbation calculation to order $m$ all terms containing $\epsilon^{l}$ with $l>m$ are discarded, while the remaining terms containing the same power of $\epsilon$ are grouped together, and the equations are solved power by power. To carry out the calculation the velocity $v(x, y)$ is written as

$$
\begin{aligned}
v(x, y) & =v(x[\rho, \theta], y[\rho, \theta]) \\
& =v^{(0)}(\rho, \theta)+\epsilon v^{(1)}(\rho, \theta)+\epsilon^{2} v^{(2)}(\rho, \theta)+\cdots .
\end{aligned}
$$

Likewise, the Laplacian operator in Eq. (3) must be expressed in terms of $\rho, \theta$, and $\epsilon$. The starting point of this transformation is the transformation of the gradients

$$
\begin{gathered}
\partial_{r}=\left(\partial_{r} \rho\right) \partial_{\rho}+\left(\partial_{r} \theta\right) \partial_{\theta}, \\
\partial_{\phi}=\left(\partial_{\phi} \rho\right) \partial_{\rho}+\left(\partial_{\phi} \theta\right) \partial_{\theta} .
\end{gathered}
$$

The derivatives $\left(\partial_{r} \rho\right),\left(\partial_{r} \theta\right),\left(\partial_{\phi} \rho\right)$, and $\left(\partial_{\phi} \theta\right)$ are obtained from the inverse transformation of Eqs. (17a) and (17b). The expansion in Eq. (19) can now be inserted into Eq. (3) and using the derivatives, Eqs. (20a) and (20b), we can carry out the perturbation scheme. The calculation of the velocity field to fourth order is straightforward, but tedious. With the velocity field at hand we can calculate the flow rate and from Eq. (4) we get

$$
\alpha=8 \pi\left[1+2(k-1) \epsilon^{2}+\frac{47-78 k+36 k^{2}-4 k^{3}}{8} \epsilon^{4}\right]+\mathcal{O}\left(\epsilon^{6}\right),
$$

where we have used the exact result $\mathcal{A}=\left(1+\frac{1}{2} \epsilon^{2}\right) \pi a^{2}$ for the area. The result only involves even powers of $\epsilon$ since $\epsilon \rightarrow$ $-\epsilon$ is equivalent to a shape rotation, which should leave $\alpha$ invariant. From a perturbative calculation of the perimeter $\mathcal{P}$ we get the following expression for $\mathcal{C}$ to second order in $\epsilon$, 


$$
\mathcal{C}=4 \pi+2 \pi\left(k^{2}-1\right) \epsilon^{2}
$$

Since $\alpha$ is also quadratic in $\epsilon$ this means that $\alpha$ depends linearly on $\mathcal{C}$ to fourth order in $\epsilon$,

$$
\alpha(\mathcal{C})=\frac{8}{1+k} \mathcal{C}-8 \frac{3-k}{1+k} \pi+\mathcal{O}\left(\epsilon^{4}\right) .
$$

Note that although derived for $k>2$ this expression coincides with that of the ellipse, Eq. (9), for $k=2$. Comparing Eq. (21) (to second order in $\epsilon$ ) with exact numerics we find that for $\epsilon$ up to 0.4 the relative error is less than $0.2 \%$ and $0.5 \%$ for $k=2$ and $k=3$, respectively.

\section{DISCUSSION AND CONCLUSION}

We have considered pressure-driven, steady-state Poiseuille flow in straight channels with various shapes, and found a close-to-linear relation between $\alpha$ and $\mathcal{C}$. Since the hydraulic resistance is $R_{\text {hyd }} \equiv \alpha R_{\text {hyd }}^{*}$, we conclude that $R_{\text {hyd }}$ depends linearly on $\mathcal{C} R_{\text {hyd }}^{*}$. Different classes of shape all display this linear relation, but the coefficients are nonuniversal. However, for each class only two points need to be calculated to fully specify the relation for the entire class. The difference is due to the smoothness of the boundaries. The elliptical and harmonic-perturbed classes have boundaries without any cusps whereas the rectangular and triangular classes have sharp corners. The overall velocity profile tends to be convex and maximal near the center-of-mass of the channel (see Fig. 1). If the boundary is smooth, the velocity in general goes to zero in a convex parabolic manner whereas a concave parabolic dependence is generally found if the boundary has a sharp corner (this can be proved explicitly for the equilateral triangle [4]). Since the concave drop is associated with a region of low velocity compared to the convex drop, geometries with sharp changes in the boundary tend to have a higher hydraulic resistance compared to smooth geometries with equivalent cross-sectional area.

We believe that the explicit and simple link between $R_{\text {hyd }}$ and $\mathcal{C}$ is an important observation since at the same time $\mathcal{C}$ is also central to the strength and effectiveness of various surface-related phenomena. We note that in micro-channels the flow properties and electrokinetic phenomena may be somewhat connected and substantial deviations from classical Poiseuille flow have been reported recently (see Ref. [7] and references therein). Nevertheless, our observation is an important first step with relevance to the use of micro-fluidic channels in lab-on-a-chip applications. Furthermore, our results allow for an easy evaluation of the hydraulic resistance for elliptical, rectangular, and triangular cross sections with the geometrical measure $\mathcal{C}$ being the only input parameter.

\section{ACKNOWLEDGMENTS}

We thank J. Kutter for stimulating discussions. N. A. M. and F. O. are supported by The Danish Technical Research Council (Grants No. 26-03-0073 and No. 26-03-0037).
[1] Microsystem Engineering of Lab-on-a-Chip Devices, edited by O. Geschke, H. Klank, and P. Telleman (Wiley-VCH Verlag, Weinheim, 2004).

[2] G. H. W. Sanders and A. Manz, Trends Analyt. Chem. 19, 364 (2000).

[3] J. Bogaert, R. Rousseau, P. Van Hecke, and I. Impens, Appl. Math. Comput. 111, 71 (2000).

[4] L. D. Landau and E. M. Lifshitz, Fluid Mechanics, 2nd ed.,
Vol. 6 of Landau and Lifshitz, Course of Theoretical Physics (Butterworth-Heinemann, Oxford, 1987).

[5] F. M. White, Fluid Mechanics (McGraw-Hill, New York, 2003).

[6] E. W. Weisstein, in MathWorld-Wolfram Web Resource (Wolfram Research, Inc., Champaign, IL, 1999), http:// mathworld.wolfram.com/Triangle.html

[7] D. J. Phares and G. T. Smedley, Phys. Fluids 16, 1267 (2004). 